To appear in The Astrophysical Journal, 492.

\title{
The Near-Infrared Number Counts and Luminosity Functions of Local Galaxies
}

\author{
Gyula P. Szokoly \\ Mark U. Subbarao \\ Andrew J. Connolly \\ Department of Physics and Astronomy, The Johns Hopkins University, Baltimore, MD 21218, \\ USA \\ and \\ Bahram Mobasher \\ Astrophysics Group, Blackett Laboratory, Imperial College, Prince Consort Road, London SW7 \\ 2BZ, U.K.
}

\begin{abstract}
This study presents a wide-field near-infrared (K-band) survey in two fields; SA 68 and Lynx 2. The survey covers an area of $0.6 \mathrm{deg} .^{2}$, complete to $\mathrm{K}=16.5$. A total of 867 galaxies are detected in this survey of which 175 have available redshifts. The near-infrared number counts to $K=16.5$ mag. are estimated from the complete photometric survey and are found to be in close agreement with other available studies. The sample is corrected for incompleteness in redshift space, using selection function in the form of a Fermi-Dirac distribution. This is then used to estimate the local near-infrared luminosity function of galaxies. A Schechter fit to the infrared data gives: $\mathrm{M}_{K}^{*}=-25.1 \pm 0.3, \alpha=-1.3 \pm 0.2$ and $\phi^{*}=(1.5 \pm 0.5) \times 10^{-3} \mathrm{Mpc}^{-3}$ (for $\mathrm{H}_{0}=50$ $\mathrm{Km} / \mathrm{sec} / \mathrm{Mpc}$ and $\left.\mathrm{q}_{0}=0.5\right)$. When reduced to $\alpha=-1$, this agrees with other available estimates of the local IRLF. We find a steeper slope for the faint-end of the infrared luminosity function when compared to previous studies. This is interpreted as due to the presence of a population of faint but evolved (metal rich) galaxies in the local Universe. However, it is not from the same population as the faint blue galaxies found in the optical surveys. The characteristic magnitude $\left(M_{K}^{*}\right)$ of the local IRLF indicates that the bright red galaxies $\left(M_{K} \sim-27\right.$ mag.) have a space density of $\leq 5 \times 10^{-5}$ $\mathrm{Mpc}^{-3}$ and hence, are not likely to be local objects.
\end{abstract}

Subject headings: surveys - cosmology: observations - galaxies: photometry - infrared: galaxies 


\section{Introduction}

The change in the intrinsic properties of galaxies as a function of lookback time provides a sensitive probe of the theories of formation of galaxies. A detailed knowledge of this is also required for interpreting the deep counts of galaxies and hence, exploring the underlying geometry of the Universe. At the optical, far-infrared and radio wavelengths, however, the observed properties of galaxies are sensitive to the morphological types, star formation history and dust content, making it difficult to disentangle the effects of evolution and cosmology at these passbands. The exception is the near-infrared wavelength $(2.2 \mu \mathrm{m})$ which is less affected by the evolutionary processes (due to the dominance of the near-infrared light by old, near-solar mass stars) and can be more securely modeled. Also, at these wavelengths the galaxy spectral energy distributions are similar for all galaxy types, leading to similar K-corrections for all galaxies. Therefore, the near-infrared number-magnitude counts of galaxies are less affected by the mix of galaxy types or uncertainties due to evolution, compared to other wavelengths. However, the interpretation of the infrared counts requires knowledge of the faint-end slope of the local infrared luminosity function (IRLF).

There are other independent studies, also requiring knowledge of the IRLF of galaxies. Due to type dependence of the optical LF (Efstathiou et al. 1988) and existence of a density-morphological type relation for galaxies, the optical LF is expected to be environmental-dependent. Indeed, recent spectroscopic studies of field galaxies have shown the existence of a population of massive, star-forming galaxies (Cowie et al. 1995; Cowie et al. 1996) in the field, not present in clusters (Trentham 1997). The IRLF is not sensitive to such star-forming galaxies, which affect the bright-end of the optical LF, and hence, is likely to have a universal form. Further, this also gives the integrated star formation in the local Universe.

Previous studies of the IRLFs of galaxies either depend on surveys selected at optical wavelengths (Mobasher et al. 1993) or, are mainly pencil beam surveys, only covering small solid angles (Cowie et al. 1996; Glazebrook et al. 1994). Also, the only study of the morphological type dependence of the IRLF (Mobasher et al. 1993) is biased against early-type galaxies (due to the optical selection of galaxies) and has too few objects of a given type. Moreover, since this sample is optically selected, it is not possible to convert it to an infrared limited survey because of the changes in type mix (i.e. color) with redshift. Therefore, it can only constrain the bright-end of the IRLF. Recently, a wide-angle near-infrared survey was performed (Gardner et al. 1997) to a limit of $\mathrm{K}=15$ magnitude. This is, however, too shallow to constrain the faint-end slope of the local IRLF with the statistics becoming very poor fainter than $M_{K}=-23.5+5 \log \left(H_{0} / 50\right)$ mag (ie. one magnitude below $M^{*}$ ), where the disagreement between different measurements of the IRLFs becomes the largest.

To overcome these problems, we have carried out a medium deep near-infrared survey, covering a large area and complete to $K \sim 16$ mag. The fields were selected to have optical multicolor data, with redshifts available for a sub-sample of galaxies. In this first paper of a series, we introduce this near-infrared selected survey. Using published redshifts, we then construct the 
local IRLF of galaxies and compare it with other, independent measurements. A study of the morphological type-dependence of the IRLF, color-magnitude relations for field galaxies and the bivariate optical/infrared LF will be presented in following papers.

In section 2 we present the observations and data reduction. Section 3 explains source detection and photometry. The astrometry and construction of the infrared catalogue are discussed in section 4. The number-magnitude counts are presented in section 5. Section 6 explores the completeness of the sample. The infrared luminosity function is constructed in section 7 . Finally, our conclusions are summarized in section 8 .

\section{Observations and Data Reduction}

The near-infrared observations presented here, were performed using the IRIM camera on the $1.3 \mathrm{~m}$ telescope at KPNO. This instrument is a NICMOS 3 array $(\mathrm{HgCdTe})$ with $256 \times 256$ pixels. The pixel scale of $1.96^{\prime \prime}$ corresponds to a field of view of $8.3^{\prime} \times 8.3^{\prime}$. This configuration enables us to undertake a wide field survey with relatively little cost in observation time. The observations were carried out in $K_{s}$ filter $(\lambda=2.15 \mu \mathrm{m} ; \Delta \lambda=0.33 \mu \mathrm{m})$. This filter is not the $\mathrm{K}^{\prime}$ filter described by Wainscoat and Cowie (1992) but the K-short filter also used by the 2MASS survey - unfortunately the IRIM instrument manual refers to it as K'. The filter cuts off about $0.1 \mu \mathrm{m}$ bluer than the standard $\mathrm{K}$ filter. This significantly reduces the thermal emission resulting in a decrease of approximately $25 \%$ in the sky background over the standard $\mathrm{K}$ filter.

Two fields were selected for near-infrared observations, Lynx-2 $\left(8^{h} 41^{m} 43.7^{s}, 44^{\circ} 46^{\prime} 42^{\prime \prime}, 1950\right)$ and $S A-68\left(0^{h} 14^{m} 53^{s}, 15^{\circ} 36^{\prime} 48^{\prime \prime}, 1950\right)$. The fields were selected to have existing multicolor photometric $\left(U B_{J} R_{F} I_{N}\right)$ and spectroscopic data (Munn et al. 1997). The near-infrared observations were carried out by dithering around each pointing position. Using an offset of 20 arcsec and an exposure time of 1 minute (20 sec. with 3 co-adds), a total of 5 exposures were taken, corresponding to an integration time of 5 minutes per pointing. This scheme was chosen to avoid cosmic rays and bad pixels. The dithered pointings were used to estimate the sky background and to construct flatfields. This dithering pattern was repeated a total of 36 times to produce a mosaic of each field. Small overlaps between the mosaic elements were incorporated into the dither pattern in order to check the internal consistency of the photometry and astrometry. Using an integration time of 5 minutes, we reach a formal magnitude limit of $K_{s}=17$ for a $5 \sigma$ detection in an 5 arcsec aperture. A total area of about 0.8 square degree was covered in both fields to a depth of $K_{s}=17$. The overlap between the optical and near-infrared survey is about 0.6 square degree.

The data were reduced using standard IRAF routines. After dark-subtraction, each exposure was flatfielded using the following three steps: Initially the dome flat was used to correct the data frames for the large-scale sensitivity variance of the detector. The flatfielded data frames were then corrected for changes in the instrument response (which was found to vary slightly over a time scale of a few minutes) by dividing them by the sky flats, constructed by median filtering 
5-10 successive data frames. Finally, the small background gradients present on the images were estimated and removed by creating a median filtered image with a very large box size and subtracting this from the original data frames.

Bad pixels were removed by deriving the average signal and variance for each pixel over the whole night and identifying those pixels that were greater than (hot pixels) or less than (non-responding pixels) $5 \sigma$ above or below the mean. Those pixels with a large variance were identified as being due to variable sensitivity (i.e. noisy pixels) and were excluded.

The final frames were registered to a common coordinate system using bright stars, not affected by bad pixels, in the frame. The measured uncertainties of the centroiding of the bright stars was 0.1-0.2 pixels. Due to the undersampling of our data and the large number of bad pixels, image shifts were done using linear interpolation after excluding each of the identified bad pixels. This procedure conserves the flux (which was our main goal) and maximizes the signal-to-noise ratio. However, it does increase the intrinsic PSF of the resultant images by about $40 \%$. The five exposures, covering the same area, were then averaged and trimmed to construct the final images. Standard photometric calibrations were derived using the UKIRT faint standards (Casali and Hawarden 1992). The observations are estimated to have an intrinsic photometric uncertainty of approximately 0.06 magnitude in the zeropoint.

\section{Source Detection and Photometry}

The source detection was performed using the SExtractor package (Bertin and Arnouts 1996). Objects were identified using a Gaussian detection kernel with FWHM of $3^{\prime \prime}$. A detection limit was set such that the objects have a minimum of 5 pixels $1.5 \sigma$ above the level of the the sky background (after convolution). This corresponds to a surface brightness of $19.7 \mathrm{mag} \operatorname{arcsec}^{-2}$. For all detected objects, two sets of magnitudes were measured, an aperture magnitude over a radius of $5^{\prime \prime}$ and a Kron magnitude with a Kron parameter of 2.5 (Kron 1980). Due to the large pixel scale and the relatively small intrinsic sizes of the galaxies at $K_{s}=15$, the difference between the aperture and Kron magnitudes were found to be negligible for the majority of our sample. At the brightest magnitude limit considered in our survey, $K_{s}=13$, the galaxy sizes exceed the aperture size and hence, are better represented by the Kron magnitudes. For the subsequent analysis in this study, we use the Kron magnitudes. For the adopted Kron parameter, these roughly correspond to the total magnitudes of galaxies. No shape parameters (e.g. second moments) were estimated from the near-infrared images because of the poor spatial resolution and the existence of higher resolution optical data.

From the selection function derived for the near-infrared data we estimate that our $5 \sigma$ detection limit is 17.0 magnitude and our completeness limit is $K_{s}=16.5$. To determine the internal accuracy of our photometry, those sources detected in the overlapping regions are compared in Fig. 1. The rms dispersion of the one-to-one correlation is 0.15 magnitude at the 
completeness limit and 0.3 magnitude at the detection limit. These are taken as the internal uncertainties in our infrared photometry. The external photometric accuracy is estimated by comparing, in Fig. 1, the $K_{s}$ band magnitudes for those galaxies in common between the present study and those by Bershady et al. (1994). This gives a larger rms scatter of 0.2 mag which is, in part, due to the fact that we are comparing our Kron magnitudes with the aperture magnitudes measured by Bershady (with associated aperture corrections). The spatial resolution of our data prevents an analysis of the Bershady data using matched apertures. It is encouraging, however, that we detect no significant zero point offset or correlation that could suggest systematic errors in either study. For the rest of this paper we assume an internal photometric accuracy of 0.15 mag.

\section{Astrometry and Comparison with the Optical Catalogue}

As noted above, the large pixel scale of the near-infrared detector here, limits our ability to perform accurate star galaxy separation (essentially all but the largest objects detected are point-like). However, for each of the survey fields, deep independent multicolor surveys exist, which can be used to classify the near-infrared detections (Koo 1986; Kron 1980). This requires accurate astrometric calibration of our detected sources.

The astrometry was carried out in two steps. Initially, approximate astrometric offsets were determined by identifying stars on each frame from the Guide Star Catalog (GSC; Lasker et al. 1990; Russell et al. 1990; Jenkner et al. 1990). This resulted in 2-6 stars per frame. Averaging over all the frames, we found the overall deviation from a zero-distortion focal plane negligible. An astrometric zero-point for each frame was determined from the GSC stars. Using this crude calibration, the near-infrared data were paired up with the optical catalogs by searching within a $10^{\prime \prime}$ radius around each detected object. From the paired data, we extract isolated sources (i.e. those near-infrared objects with only one optical counterpart within the search radius) and rederive a more accurate astrometric solution. While the astrometric solution for each frame can be established rather accurately $\left(0.1^{\prime \prime}-0.2^{\prime \prime}\right)$, the uncertainty in astrometry for individual objects remains large $\left(\sim 1.8^{\prime \prime}\right)$, due to the large PSF.

We construct an optical-near-infrared catalogue of all the detected objects in the overlapping region, by extending the technique of Sutherland and Saunders (1992) to use both positional and magnitude information. The simplest method to identify the same object in different catalogs is to estimate the maximum positional uncertainty and pair-up the detected objects with separations smaller than this threshold. This technique works well for surveys where the average astrometric uncertainty is much smaller than the typical separation between the objects. However, for moderately dense fields, this method does not produce reliable catalogs as shown for our dataset in Figure 3a. The dashed line is the number of near-infrared detections without an optical counterpart and indicates that a tolerance level of $\sim 2$ normalized distance units is required (normalized distance is $\sqrt{\left(d_{1} / \sigma_{1}\right)^{2}+\left(d_{2} / \sigma_{2}\right)^{2}}$, where $d_{1}$ and $d_{2}$ are the angular positional differences along the two axes with $\sigma_{1}$ and $\sigma_{2}$ their mean respective uncertainties). At this level, 
about $25 \%$ of the detections have multiple optical counterparts (dotted line). The solid line shows the number of cases where this simple method results in unique matches. This cuts-off rather sharply, indicating that this identification is not too reliable.

To eliminate multiple hits within the matched catalogs the usual practice is to pick the closest object from the optical catalog. One can assign a likelihood to this identification

$$
L=\frac{q(m) f(x, y)}{n(m)}
$$

where $q(m)$ is the probability distribution in magnitude, $f(x, y)$ describes the distribution of positional uncertainties and $n(m)$ is the surface density of objects of magnitude $m$. This method does not take into account the local information. For isolated objects, the match is likely to be genuine even if the positional uncertainty is large, while in a crowded area multiple objects can be very close, making the identification unreliable (even though the likelihood is high). Following Sutherland and Saunders (1992), we address this problem by defining a new local quantity, the reliability, as

$$
R=\frac{L_{i}}{\sum_{j} L_{j}+(1-Q)}
$$

where $L_{i}$ is the likelihood of an identification, as defined above, $L_{j}$ are the likelihoods of other candidates and $Q$ is the probability that the identification is possible (i.e. both catalogs contain the object). This formalism properly takes into account the existence of other identified candidates.

In the present study, we extend this technique to include positional and magnitude information. First, we identify isolated objects (sources with no other detection within a $10^{\prime \prime}$ radius) present in both the catalogs. Since the infrared survey is not as deep as its optical counterpart, this subset, in practice, contains only genuine identifications. From these data we then derive a correlation between the near-infrared $\left(K_{s}\right.$-band) and optical detections by fitting the function $K^{*}\left(U, B_{J}, R_{F}, I_{N}\right)$ to a second degree polynomial (Connolly et al. 1995). To allow for differences in optical photometry between the two fields, this procedure was done independently for each field. The correlation between the predicted and measured K-band magnitudes is shown in Fig. 2. The mean residual uncertainty for both fields is 0.45 mag. and the error distribution is Gaussian with no outlying points. Since about $40 \%$ of the galaxies are isolated, we do not expect more than a few (at most five) galaxies with unusual K-magnitude in the full survey.

Using the fit $K^{*}\left(U, B_{J}, R_{F}, I_{N}\right)$, a normalized distance metric is then defined as

$$
\rho=\sqrt{\frac{\left(\alpha_{K}-\alpha_{0}\right)^{2}}{\sigma_{\alpha}^{2}}+\frac{\left(\delta_{K}-\delta_{0}\right)^{2}}{\sigma_{\delta}^{2}}+\frac{\left(K-K^{*}\right)^{2}}{\sigma_{K}^{2}}}
$$

where $\sigma_{\alpha}, \sigma_{\delta}, \sigma_{K}$ are the uncertainties in the spatial and color dimensions. Since the distributions in all three directions are Gaussian and independent, the likelihood functions are then calculated as

$$
L=\frac{f(\alpha, \delta, K)}{n(K)} \approx Q(<K) \frac{e^{-\rho^{2} / 2}}{2 \pi \sigma_{\alpha} \sigma_{\delta} \sigma_{K}}
$$


where $n(K)$ is the density of optical detections and $Q(<K)$ the probability that an object of magnitude $\mathrm{K}$ has an optical counterpart (in this case $Q(<K) \approx 1$ for the infrared catalogue). Finally, the reliability of the match is defined as

$$
R_{j}=\frac{L_{j}}{\Sigma_{i} L_{i}+(1-Q)} \approx \frac{L_{j}}{\Sigma_{i} L_{i}}
$$

This technique is used to cross-compare the near-infrared and optical catalogs and as demonstrated in Figure 3b, it considerably improves the match between the two surveys. The ratio of number of objects uniquely matched to the number of objects with multiple matches is, at all distances, greater than that derived when using only the positional information. Therefore, including color information greatly reduces the number of spurious pairs, resulting in a much more reliable catalog.

For all the matched objects, the most reliable optical detection was entered into the final catalogue. The calculated reliabilities, in most cases, were found to be close to 1 . For each object with an optical counterpart, we adopt the classification (star or galaxy) as defined in the optical catalog. An area of $0.8 \mathrm{deg}^{2}$ was surveyed in the near-infrared, of which $0.6 \mathrm{deg}^{2}{ }^{2}$ overlaps with the multicolor optical surveys. Over this area, a total of 871 objects were detected in the near-infrared survey, of which 867 have optically identified counterparts. The 4 objects without an optical counterpart are likely to correspond to faint spurious detections.

The final infrared catalogue contains 867 galaxies to $K_{s}=17$ mag. All these objects have optical data. The redshifts are compiled from literature for a sub-set of galaxies in the infrared catalogue, giving a total of 175 galaxies with measured redshifts (Munn et al. 1997). This sample will be used in the following sections to construct the number counts and near-infrared luminosity function of local galaxies.

Using the estimated K-band magnitudes, we verify that our survey is shallow enough such that blending of close pairs of galaxies does not significantly affect the catalog. We calculate the expected infrared magnitude for every optical galaxy around each infrared detection. We individually examine all infrared detections that have multiple optical counterparts within 10

arcsec and with $K_{\text {predicted }}<16.5$ (i.e. sufficiently bright that they would make it into the K selected sample). Within the complete photometric survey there were 10 such candidates each of which was handled correctly by the photometry package.

\section{The Near-infrared Number Counts}

The near-infrared number counts, estimated from this study, are presented in Table 1 . These are compared in Fig. 4 and Table 2 with the counts from other independent studies as compiled by Gardnet et al. (1993). The comparison between the various studies is made over the magnitude range covered in this study $\left(K_{s}=13-16.5\right.$ mag.). In all the counts in Figure 4, the uncertainties are estimated using the technique developed by Gehrels (1986). 
The counts derived from our two independent fields, SA68 and Lynx2, agree within their uncertainties over the entire magnitude range covered here. They are also in good agreement with the counts in Gardner (1996) and Huang and Cowie (1997), each covering a larger area (9.8 $\left.\operatorname{deg}^{2}\right)$ but to a shallower depth $(K<16)$. These results confirm that the present near-ir survey is complete to $K_{s}=16.5 \mathrm{mag}$. Moreover, it indicates that there is no anomaly, due to density enhancement in our fields or photometric zero-points, affecting the near-ir distribution of galaxies in this study.

Fitting a linear relation to the number-magnitude counts for $14.5<K_{s}<16.5$ we derive a slope of $0.46 \pm 0.06,0.53 \pm 0.04$ and $0.50 \pm 0.03$ for the SA68, Lynx2 and combined samples respectively. These values are lower than those derived by Huang and Cowie (1997) and Gardner (1997) from their wide field survey. They are also lower than the predictions by Huang et al. (1997) for a no evolutionary model. The shallower slope may be indicative of an overdensity of low redshift galaxies in our two fields (which is clearly seen in the redshift distribution of the Lynx2 field but not in SA68). It is more likely, however, that the variation amongst the measured slopes is an artifact of fitting linear relations over a narrow range of magnitudes.

\section{Selection Functions}

As the spectroscopic observations in this study are based on an optically selected sample, it is likely that at fainter $K_{s}$ magnitudes, the survey becomes incomplete in redshift space. Therefore, it is instructive to estimate the incompleteness in redshift as a function of $K_{s}$. Assuming a Fermi-Dirac distribution for the selection function (Sandage, Tammann and Yahil 1979),

$\left(\exp \left(\frac{m-m_{l}}{\Delta m}\right)+1\right)^{-1}$, the incompleteness (defined as the change with magnitude of the ratio of the number of galaxies with measured redshift to the total number of galaxies to a given $K_{s}$ limit), is calculated and fitted to this parametric form in Fig. 5. We find $m_{l}=15.25, \Delta m_{l}=0.80$ for SA68 and $m_{l}=14.86, \Delta m_{l}=0.54$ for Lynx2. For the luminosity function analysis in this study, we select a redshift completeness limit of $30 \%$ in Fig. 5. This corresponds to a limiting magnitude of $K_{s}=16$ and $K_{s}=15.25$ in the SA68 and Lynx2 fields respectively. These will be used as the completeness limits in the following section to construct the near-ir luminosity function. The redshift distributions for the complete sample, containing both the fields, are presented in Fig. 6 . There are a total of 110 galaxies in the two fields brighter than the above $K_{s}$-band magnitude limits in this survey. Assuming the estimated magnitude limits, a $V / V_{m}$ test (Schmidt's 1968) gives $\left\langle V / V_{m}\right\rangle=0.50 \pm 0.02$ and $\left\langle V / V_{m}\right\rangle=0.35 \pm 0.02$ for the SA68 and Lynx2 fields respectively. The smaller than expected $\left\langle V / V_{m}\right\rangle$ for the Lynx2 field is likely to be due to a cluster at $z \sim 0.05$ in this field (Fig. 6). The effect of this density enhancement on the IRLF will be explored in the next section. The redshift distribution in Figure 6 also shows that the galaxies in the infrared survey here are mainly local $(z<0.4)$ objects. 


\section{Local Near-ir Luminosity Function}

Most determinations of the luminosity function assume a parametric form (normally a Schechter form) to fit to the observed data. The shape of the luminosity function is, however, likely to be type-dependent or be affected by density enhancements or its local environment. For these reasons, and because of the presence of a cluster at $z=0.05$ in the Lynx2 field, we first use the non-parametric C-method (Lynden-Bell 1971) to assess the shape of the local near-ir luminosity function. A parametric maximum likelihood method (Sandage, Tammann and Yahi] 1979), insensitive to density enhancement, will then be used to find the best fit to the data.

Using the near-infrared K-corrections from Glazebrook et al. (1995), the K-band luminosity function, estimated from the C-method is shown in Fig. 7. The uncertainties are estimated using bootstrap re-sampling simulations. Clearly, the shape of the local infrared luminosity function here is consistent with a Schechter form (Schechter 1976). A parametric fit to the data, using the cluster-free maximum likelihood method, gives $M_{K_{s}}^{*}=-25.05+5 \log \left(H_{0} / 50\right)$ and $\alpha=-1.27$. The correlated uncertainty contours at $68 \%, 90 \%$ and $95 \%$ levels are estimated for $M_{K_{s}}^{*}$ and $\alpha$ and presented in Fig. 8. From these, we estimate $3 \sigma$ uncertainties corresponding to 0.3 mag. and 0.2 in $M_{K_{s}}^{*}$ and $\alpha$ respectively.

To explore the sensitivity of this result to the density enhancement in the Lynx2 field, we also find the luminosity function using the 'conventional' technique. This calculates the contribution from each galaxy to the volume covered by that galaxy at the apparent magnitude limit of the survey $\left(1 / \mathrm{V}_{\max }\right)$. The total contribution of galaxies in absolute magnitude intervals $\left(\sum_{i=1}^{n} 1 / V_{\max }^{(i)}\right)$ is then estimated and compared with the result from the cluster-free C-method in Figure 7. A Schechter function fit to these data, also shown in Figure 7, gives: $\mathrm{M}_{K_{s}}^{*}=-24.90$ mag., $\alpha=-1.42$ and $\phi^{*}=9.5 \times 10^{-4} \mathrm{Mpc}^{-3}$. Within the uncertainties, this is similar to the parameters estimated from the cluster-free maximum likelihood method, indicating that the presence of non-homogeneities in our sample does not significantly affect the resulting luminosity function. Therefore, the parameters from the C-method/cluster-free fit here will be taken as the values for the local near-infrared luminosity function in this study.

The normalization of the luminosity function $\left(\phi^{*}\right.$ in the Schechter formalism), not given by the cluster-free maximum likelihood technique, is estimated using three different methods. First, performing a $\chi^{2}$ minimization of the Schechter function to the results from the C-method in Figure 7. Second, using the $\phi^{*}$ value estimated from the 'conventional' technique, as presented above. Third, employing our estimate of $M_{K_{s}}^{*}$ and $\alpha$ to establish a model of the K-band number counts and normalizing this to the observed counts in Fig. 4. We adopt the normalization given by the C-method and estimate the uncertainty based on the variation in $\phi^{*}$ derived by the three methods: $\phi^{*}=(0.15 \pm 0.05) \times 10^{-2}\left(H_{0} / 50\right)^{3} \mathrm{Mpc}^{-3}$.

The IRLFs in Figure 7 are compared with a similar study by Gardner et al (1997). Their measured IRLF is based on a larger sample ( $\sim 500$ galaxies) but is less deep than the present survey (it is only complete to $K \sim 15$ mag.). Although both the bright-end and normalizations 
agree fairly closely, the faint-end of the IRLF in this study is significantly steeper. The reality of the steep faint-end slope for the local IRLF, found here, will be further investigated by measuring redshifts for the fainter galaxies in our sample, extending the completeness of our survey to $K_{s} \sim 17$ mag.

In order to avoid the correlation between $M^{*}$ and $\alpha$ affecting the fit and to compare the present luminosity function with other independent studies, we fix $\alpha=-1$ and estimate $M_{K_{s}}^{*}$. The local IRLFs, estimated by different groups, are presented in Table 3. The characteristic magnitude $\left(M_{K_{s}}^{*}\right)$ here, is transformed from $K_{s}$ to $\mathrm{K}$, using the relation in section 5. Following Glazebrook et al. (1995), we apply a correction of +0.22 mag. to Mobasher et al. (1993) measurement to account for differences in K-corrections. Also, an aperture correction of -0.3 magnitude is applied to Glazebrook et al. 1995 to convert it to the same scale as other measurements. All the estimates are corrected to $H_{0}=50 \mathrm{Km} / \mathrm{sec} / \mathrm{Mpc}$. It is clear from Table 3 that, at a given $\alpha$, the $M_{K}^{*}$ values from different methods are in close agreement. The space density of local galaxies $\left(\phi^{*}\right)$ in this study is slightly smaller than other similar measurements $(\sim 1.5)$. This will be further explored by increasing the size of our sample, extending its completeness in redshift space.

The steep faint-end slope of the local IRLF, if confirmed, will have important implications towards constraining the models for formation of nearby, low-luminosity field galaxies (ie. the mergers scenarios) since the IRLF is mainly sensitive to the mass function and not the star formation (young population) in galaxies. Also, this implies the existence of a large population of evolved, metal rich galaxies in the local Universe. The color distribution of the K-selected surveys shows that the faint blue galaxies start to contribute to the galaxy counts at about $K \sim 18$ mag. (Gardner 1995). Such near-infrared surveys will then reveal if the faint blue galaxies have an underlying population of old stars. The surveys selected in the K-band are mainly dominated by normal massive galaxies. Therefore, the characteristic magnitude $\left(M_{K}^{*}\right)$ found for the IRLF here implies that the very red galaxies with $M_{K} \sim-27$ mag. (Egami et al. 1996; Graham and Day 1996) have a space density of $\leq 5 \times 10^{-5} \mathrm{Mpc}^{-3}$ and hence, are not likely to be local objects $(z<0.4)$.

We are currently completing the redshift measurements for the fainter galaxies $\left(K_{s}>16\right)$ in the present survey. This will be used to further constrain the faint-end slope of the 'local' infrared luminosity function by improving the statistical significance of the sample and to explore its morphological type dependence.

\section{Conclusions}

In this study we carried out a wide-angle near-infrared galaxy survey in two fields, SA68 and Lynx2. The survey is complete to $K_{s}=16$ (SA68) and $K_{s}=15.25$ (Lynx2) and covers a total area of $0.6 \mathrm{deg} .{ }^{2}$. Matching the near-infrared detections with existing optical multicolor surveys we derive a catalog of 867 galaxies. Of these, 175 have available redshifts. Employing the complete 
photometric survey, the near-infrared number-magnitude counts are estimated to $K \sim 16.5$ mag. and found to be in agreement with other independent measurements.

Correcting the spectroscopic samples for incompleteness in redshift space, using selection functions in the form of Fermi-Dirac distribution, the luminosity function has been derived for local galaxies. Applying a parametric Schechter fit we find $M_{K}^{*}=-25.09 \pm 0.3, \alpha=-1.27 \pm 0.2$ and $\phi^{*}=(0.15 \pm 0.05) \times 10^{-2} \mathrm{Mpc}^{-3}$ (for $\mathrm{H}_{0}=50 \mathrm{Km} / \mathrm{sec} / \mathrm{Mpc}$ and $\left.\mathrm{q}_{0}=0.5\right)$. When reduced to $\alpha=-1$, this agrees with other available estimates of the local IRLF.

The most important limitation of our survey is that the redshift survey is optically selected. Indeed we see a systematic, color dependent incompleteness at $K_{s}>14.5$ in the Lynx-2 field and $K_{s}>15$ in the SA-68. To estimate the effect of this approximation, we used the simulated catalogs produced by Gronwall and Koo (1995). We calculate the K-band luminosity function for an ideal, 100 percent complete, K-selected catalog and for an optically selected redshift catalog with a selection function somewhat more conservative than that of the KPGRS data $(R<18.5)$. We found that the two calculated luminosity functions are identical, within the uncertainties, up to $M_{K_{s}}=-22.5$. For $M_{K_{s}}>-21.5$ the optically selected sample systematically overestimates the amplitude of the luminosity function. We, therefore, limit our analysis to $M_{K_{s}}=-22.5$ for the current data set.

The faint-end slope of the IRLF here is steeper than previous studies. This implies the presence of a population of faint but evolved (metal rich) galaxies in the local Universe. Comparison with the flat faint-end slope of the optical luminosity function reveals that this is unlikely to be the same population as the faint blue galaxies found in the optical surveys. The characteristic magnitude $\left(M_{K}^{*}\right)$ of the local IRLF indicates that the bright red galaxies $\left(M_{K} \sim-27\right.$ mag.) have a space density of $\leq 5 \times 10^{-5} \mathrm{Mpc}^{-3}$ and hence, are not likely to be local objects.

We thank Mark Dickinson and Alex Szalay for useful discussions about the analysis and interpretation of the near-infrared data. We are grateful to David Koo, Richard Kron, Jeffrey Munn, Steven Majewski, Matthew Bershady, and John Smetanka for pre-publication access to the KPGRS catalogs. 
Table 1. $\quad K$-Band Differential Number Counts

\begin{tabular}{|c|c|c|c|c|c|c|}
\hline Survey & K & Raw $N$ & Low & High & Area $\left(\operatorname{arcmin}^{2}\right)$ & $\mathrm{N} / \mathrm{mag} / \mathrm{deg}^{2}$ \\
\hline \multirow[t]{8}{*}{ SA-68 field } & 13.0 & 1 & 0.17 & 3.3 & 667 & 10.8 \\
\hline & 13.5 & 1 & 0.17 & 3.3 & & 10.8 \\
\hline & 14.0 & 2 & 0.71 & 4.6 & & 21.6 \\
\hline & 14.5 & 11 & 7.7 & 15 & & 119 \\
\hline & 15.0 & 26 & 21 & 32 & & 281 \\
\hline & 15.5 & 39 & 33 & 46 & & 421 \\
\hline & 16.0 & 69 & 61 & 78 & & 745 \\
\hline & 16.5 & 120 & 109 & 132 & & 1295 \\
\hline \multirow[t]{13}{*}{ Lynx-2 field } & 10.5 & 1 & 0.17 & 3.3 & 1519 & 4.7 \\
\hline & 11.0 & 0 & 0 & 1.8 & & 0 \\
\hline & 11.5 & 1 & 0.17 & 3.3 & & 4.7 \\
\hline & 12.0 & 5 & 2.8 & 8.4 & & 24 \\
\hline & 12.5 & 1 & 0.17 & 3.3 & & 4.7 \\
\hline & 13.0 & 6 & 3.6 & 9.6 & & 28 \\
\hline & 13.5 & 6 & 3.6 & 9.6 & & 28 \\
\hline & 14.0 & 7 & 4.4 & 11 & & 33 \\
\hline & 14.5 & 26 & 21 & 32 & & 123 \\
\hline & 15.0 & 42 & 36 & 50 & & 199 \\
\hline & 15.5 & 81 & 72 & 91 & & 384 \\
\hline & 16.0 & 160 & 147 & 174 & & 758 \\
\hline & 16.5 & 270 & 254 & 287 & & 1279 \\
\hline \multirow[t]{8}{*}{ Combined fields } & 13.0 & 7 & 4.4 & 11 & 2185 & 23 \\
\hline & 13.5 & 7 & 4.4 & 11 & & 23 \\
\hline & 14.0 & 9 & 6.1 & 13 & & 30 \\
\hline & 14.5 & 37 & 31 & 44 & & 122 \\
\hline & 15.0 & 68 & 60 & 77 & & 224 \\
\hline & 15.5 & 120 & 109 & 132 & & 395 \\
\hline & 16.0 & 229 & 214 & 245 & & 755 \\
\hline & 16.5 & 390 & 370 & 410 & & 1285 \\
\hline
\end{tabular}

Listed are the field, the $K$-magnitude (center of the bin), the raw number of galaxies, the upper and lower $1 \sigma$ limits for the raw counts (Gehrels 1986), the survey area and the number per magnitude per square degree. 
Table 2. $\quad K$-Band Differential Number Counts

\begin{tabular}{|c|c|c|c|c|c|c|}
\hline Survey & $\mathrm{K}$ & Raw $N$ & Low & High & Area $\left(\operatorname{arcmin}^{2}\right)$ & $\mathrm{N} / \mathrm{mag} / \mathrm{deg}^{2}$ \\
\hline \multirow[t]{3}{*}{ Gardner '93 (HWS) } & 12.5 & 5 & 2.8 & 8.4 & 5690 & 0.0009 \\
\hline & 13.5 & 23 & 18 & 29 & & 0.004 \\
\hline & 14.5 & 124 & 113 & 136 & & 0.02 \\
\hline \multirow[t]{9}{*}{ Gardner '93 (HMWS) } & 12.75 & 2 & 0.71 & 4.6 & 582.03 & 24.7 \\
\hline & 13.25 & 0 & 0 & 1.8 & & 0 \\
\hline & 13.75 & 1 & 0.17 & 3.3 & & 12.4 \\
\hline & 14.25 & 3 & 1.4 & 5.9 & & 37.1 \\
\hline & 14.75 & 11 & 7.7 & 15 & & 136 \\
\hline & 15.25 & 22 & 17 & 28 & & 272 \\
\hline & 15.75 & 45 & 38 & 53 & & 557 \\
\hline & 16.25 & 89 & 80 & 99 & & 1100 \\
\hline & 16.75 & 158 & 145 & 172 & & 1900 \\
\hline \multirow[t]{7}{*}{ Gardner '93 (HMDS) } & 13.75 & 2 & 0.71 & 4.6 & 167.68 & 86 \\
\hline & 14.25 & 0 & 0 & 1.8 & & 0 \\
\hline & 14.75 & 1 & 0.17 & 3.3 & & 43 \\
\hline & 15.25 & 5 & 2.8 & 8.4 & & 215 \\
\hline & 15.75 & 21 & 16 & 27 & & 902 \\
\hline & 16.25 & 28 & 23 & 34 & & 1200 \\
\hline & 16.75 & 48 & 41 & 56 & & 2060 \\
\hline \multirow[t]{4}{*}{ Glazebrook '93 } & 13.5 & 3 & 1.4 & 5.9 & 551.9 & 0.005 \\
\hline & 14.5 & 8 & 5.2 & 12 & 551.9 & 0.0145 \\
\hline & 15.5 & 62 & 54 & 71 & 551.9 & 0.112 \\
\hline & 16.5 & 168 & 155 & 182 & 551.9 & 0.304 \\
\hline \multirow[t]{12}{*}{ Gardner '96 } & 10.25 & 1 & 0.17 & 3.3 & 30750 & $6 \times 10^{-5}$ \\
\hline & 10.75 & 1 & 0.17 & 3.3 & & $6 \times 10^{-5}$ \\
\hline & 11.25 & 4 & 1.4 & 7.1 & & $2.6 \times 10^{-4}$ \\
\hline & 11.75 & 13 & 9.4 & 18 & & $8.4 \times 10^{-4}$ \\
\hline & 12.25 & 22 & 17 & 28 & & 0.0014 \\
\hline & 12.75 & 33 & 27 & 40 & & 0.0021 \\
\hline & 13.25 & 66 & 58 & 75 & & 0.0043 \\
\hline & 13.75 & 138 & 126 & 151 & & 0.0090 \\
\hline & 14.25 & 273 & 256 & 290 & & 0.0177 \\
\hline & 14.75 & 642 & 617 & 668 & & 0.0418 \\
\hline & 15.25 & 1290 & 1250 & 1330 & & 0.0839 \\
\hline & 15.75 & 2609 & 2560 & 2660 & & 0.1697 \\
\hline
\end{tabular}

Listed are the survey, the $K$-magnitude (center of the bin), the raw number of galaxies, the upper and lower $1 \sigma$ limits for the raw counts, the survey area and the number per magnitude per square degree. 
Table 3. Schechter parameters for near-infrared luminosity function

\begin{tabular}{lcrcc}
\hline \hline \multicolumn{1}{c}{ study } & $M_{K}^{*}$ & \multicolumn{1}{c}{$\alpha$} & \multicolumn{1}{c}{$\Phi^{*}$} & $\mathrm{n}$ \\
\hline This study (C-method) & -25.09 & -1.27 & $0.15 \times 10^{-2}$ & 110 \\
This study (conventional method) & -24.94 & -1.42 & $0.95 \times 10^{-3}$ & 110 \\
This study $\alpha=-1$ & -24.84 & -1 & $0.15 \times 10^{-2}$ & 110 \\
Gardner et al. 1997 & -24.87 & -1.03 & $0.22 \times 10^{-2}$ & 532 \\
Glazebrook et al. 1995 & -24.55 & -1.04 & $0.33 \times 10^{-2}$ & 98 \\
\hline Mobasher et al. 1993 & -24.88 & -1 & $0.14 \times 10^{-2}$ & 95 \\
\hline
\end{tabular}




\section{REFERENCES}

Bershady, M. A. 1995, AJ, 109, 87

Bershady, M. A.; Hereld, M., Kron, R. G., Koo, D. C., Munn, J. A.; Majewsky, S. R. 1994, AJ, 108,870

Bertin, E.; Arnouts, S. 1996 A\&AS, 117, 393

Casali, M. M. and Hawarden 1992, T. G., The JCMT-UKIRT Newsletter Vol. 4, p. 33

Connolly, A. J., Csabai, I., Szalay, A. S., Koo, D. C., Kron, R. G., Munn, J. A. 1995, AJ, 110, 2655

Cowie, L. L., Hu, E. M., Songaila, A. 1995, Nature, 377, 603

Cowie, L. L., Songaila, A., Hu, E. M., Cohen, J. G. 1996, AJ, 112, 839

Efstathiou, G.; Ellis, R. S.; Peterson, B. A. 1988, MNRAS, 232, 431

Egami, E.; Hu, E; Cowie, L.L 1996 ApJ112, 73

Gardner, J. P., 1995, ApJS, 98, 441.

Gardner, J. P. 1996, MNRAS, 279, 1157

Gardner, J. P., Cowie, L. L., Wainscoat, R. J. 1993, ApJ, 415, L9

Gardner, J. P., Sharples, R. M., Frenk, C. S., Carrasco, B. E., 1997, ApJ, 480, L99

Gardner, J. P., Sharples, R. M., Carrasco, B. E., Frenk, C. S. 1996, MNRAS, in press

Gehrels, N. 1986, ApJ, 303336

Glazebrook, K., Peacock, J. A., Collins, C. A. and Miller, L. 1994, MNRAS, 266, 65

Glazebrook, K., Peacock, J. A., Miller, J. A., Collins 1995, MNRAS, 275, 169

Graham, J.R; Day, A. 1996 ApJ471, 720

Gronwall, C.; Koo, D. C. 1993, BAAS, 182, 3002

Huang, J. S., Cowie, L. L., 1997, in preparation

Huang, J. S., Cowie, L. L., Gardner, J. P., Hu, E. M., Songaila, A., Wainscoat, R. J., 1997, ApJ, 476,12

Jenker, H., Lasker, B. M., Sturch, C. R., McLean, B. J., Shara, M. M., Russell, J. L., 1990, AJ, 99,2082 
Lasker, B. M., Sturch, C. R., McLean, B. J., Russell, J. L., Jenker, H., Shara, M. M., 1990, AJ, 99, 2019

Lynden-Bell, D., 1971, MNRAS, 155, 95.

Koo, D. C., 1986, ApJ, 311, 651.

Kron, R. G., 1980, ApJS, 43, 305.

Mobasher, B., Ellis, R. S. and Sharples, R. M. 1986, MNRAS, 223, 11

Mobasher, B., Sharples, R. M. and Ellis, R. S. 1993, MNRAS, 263, 560

Munn, J. A., Koo, D. C., Kron, R. G., Majewski, S. R., Bershady, M. A., Smetanka, J. J., 1997 ApJS, 109, 45

Russell, J. L., Lasker, B. M., McLean, B. J., Sturch, C. R., Jenker, H., 1990, AJ, 99, 2059

Sandage, A., Tammann, G.A., \& Yahil, A., 1979, ApJ, 232, 352.

Schechter, P., 1976, ApJ, 203, 297.

Schmidt,M. 1968, ApJ, 151, 393.

Sutherland W. and Saunders W. 1992, MNRAS, 259, 413.

Trentham, N. 1997, MNRAS, 286, 133.

Wainscoat, R. J.; Cowie, L. L., 1992, AJ, 103, 332. 

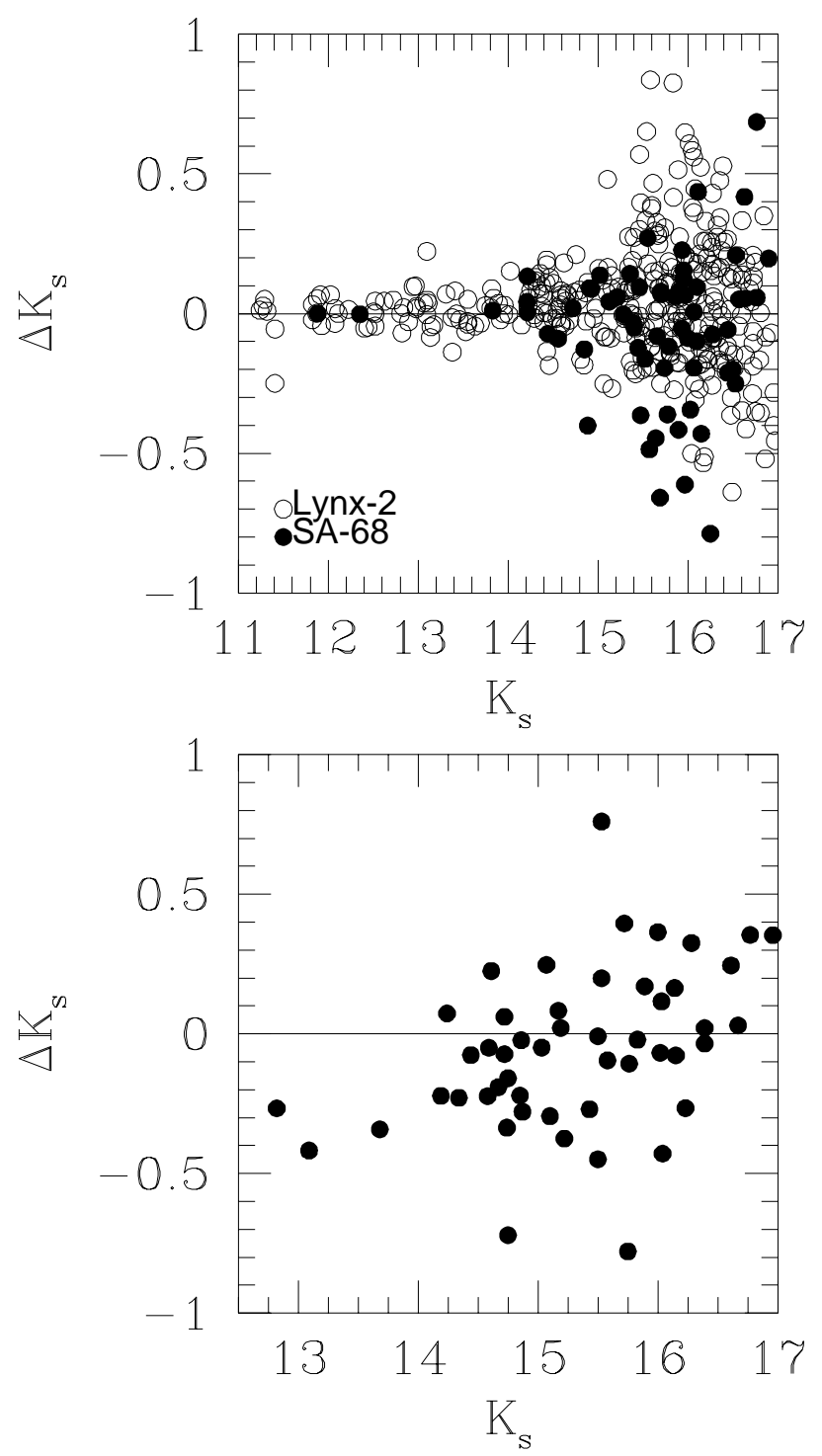

Fig. 1. - The photometric uncertainties within our data are estimated internally from a comparison between repeated observations and externally using published K-band photometry. The top panel shows the dispersion about a one-to-one correlation for those sources detected in overlap IRIM frames. At the completeness limit $\left(K_{s}=16.5\right)$ the $\mathrm{rms}$ dispersion is 0.15 magnitude. The lower panel shows a comparison between our photometry and that of Bershady (Bershady et al. 1994), $K_{s}^{(1)}$. There is no significant offset between our photometric system but due to the use of different magnitudes the rms dispersion increases to 0.2 magnitudes. 


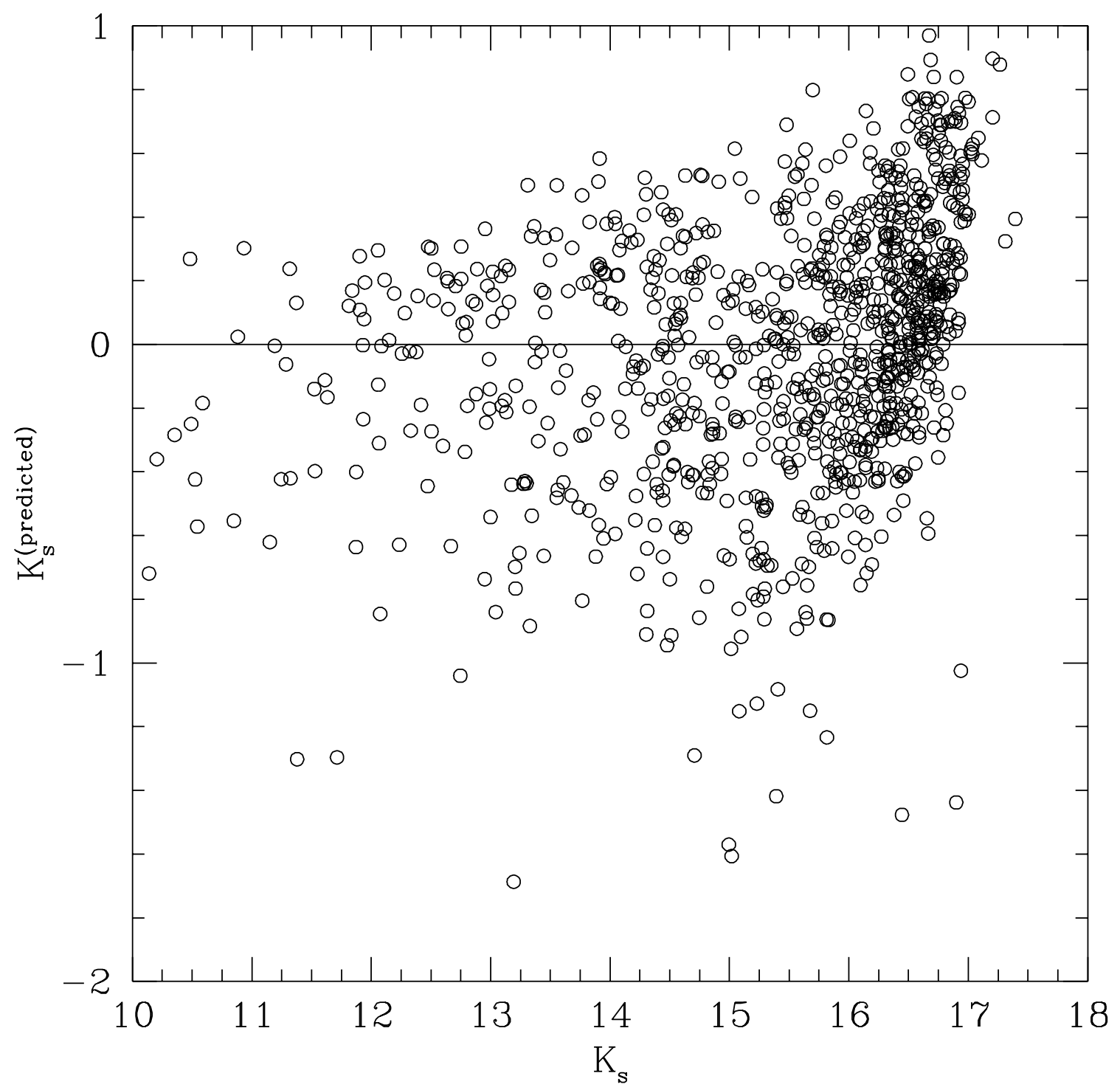

Fig. 2.- The correlation between predicted and measure $K_{s}$ magnitudes. The estimated magnitude is derived by fitting a second degree polynomial function to the optical $U, J, F, N$ photometry. The rms dispersion about this relation is 0.4 magnitudes. Using this correlation the optical and nearinfrared data were using a combined angular and magnitude distance metric. 

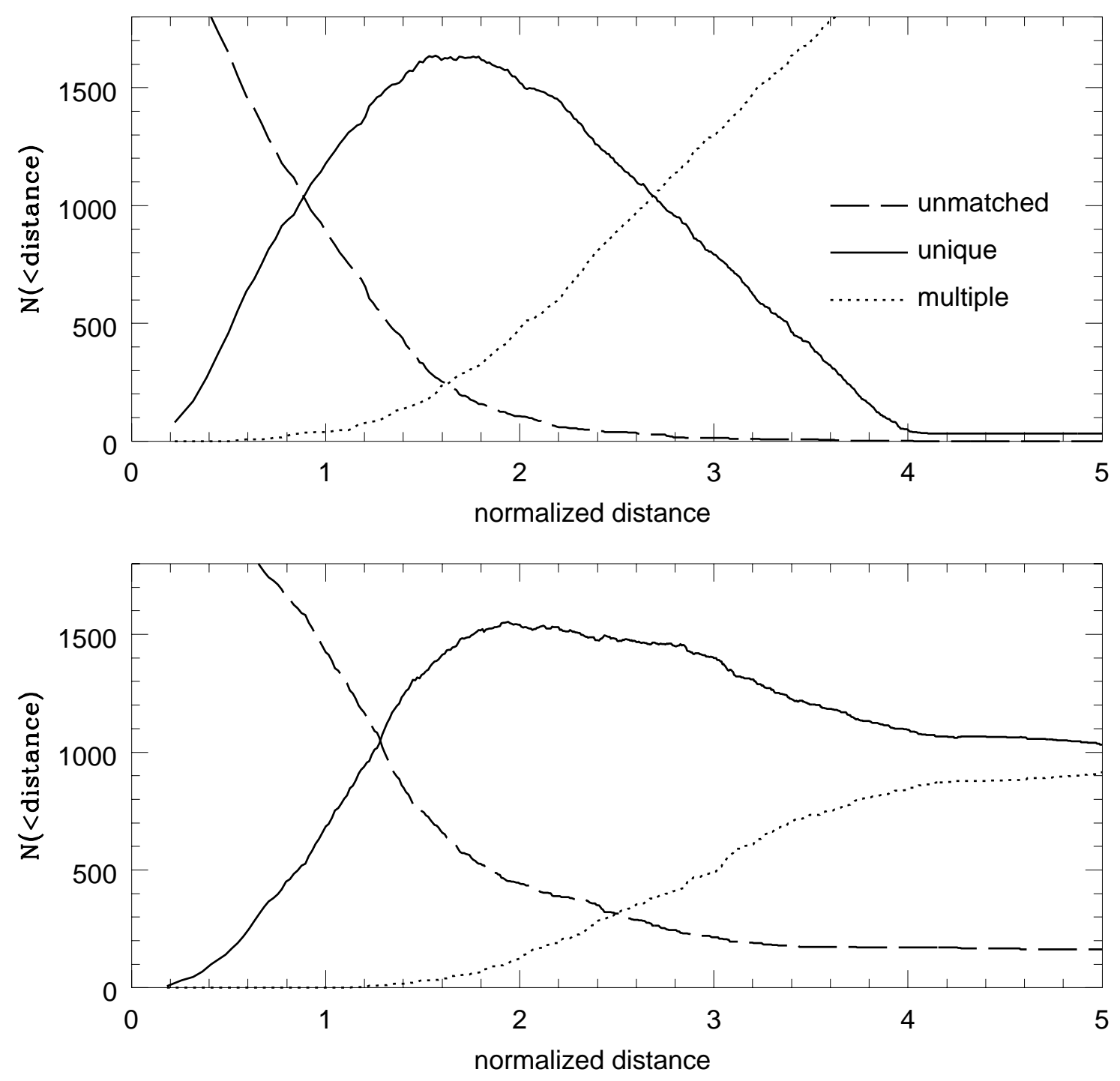

Fig. 3. - The effect of using color information to cross-id catalogs. When one uses angular distance only (top figure), the acceptance criteria is very critical. Setting the limit too low will result in the rejection of valid pairs (dashed curve). Relaxing the criteria too much results in too many ambiguous pairs (dotted line). Including the color information eliminates most of the accidental coincidences (dashed line) and drastically reduces the number of ambiguous matches. At all distances the ratio of unique to multiple matchups is larger when we incorporate color information. Further, the choice of tolerance for the metric is much less important (the solid line, the number of unique identifications dies off gently). In both cases we used the dimensionless normalized distance, $\rho=\sqrt{\frac{\left(\alpha_{K}-\alpha_{0}\right)^{2}}{\sigma_{\alpha}^{2}}+\frac{\left(\delta_{K}-\delta_{0}\right)^{2}}{\sigma_{\delta}^{2}}}$ and $\rho=\sqrt{\frac{\left(\alpha_{K}-\alpha_{0}\right)^{2}}{\sigma_{\alpha}^{2}}+\frac{\left(\delta_{K}-\delta_{0}\right)^{2}}{\sigma_{\delta}^{2}}+\frac{\left(K-K^{*}\right)^{2}}{\sigma_{K}^{2}}}$ as defined in section 4 . 


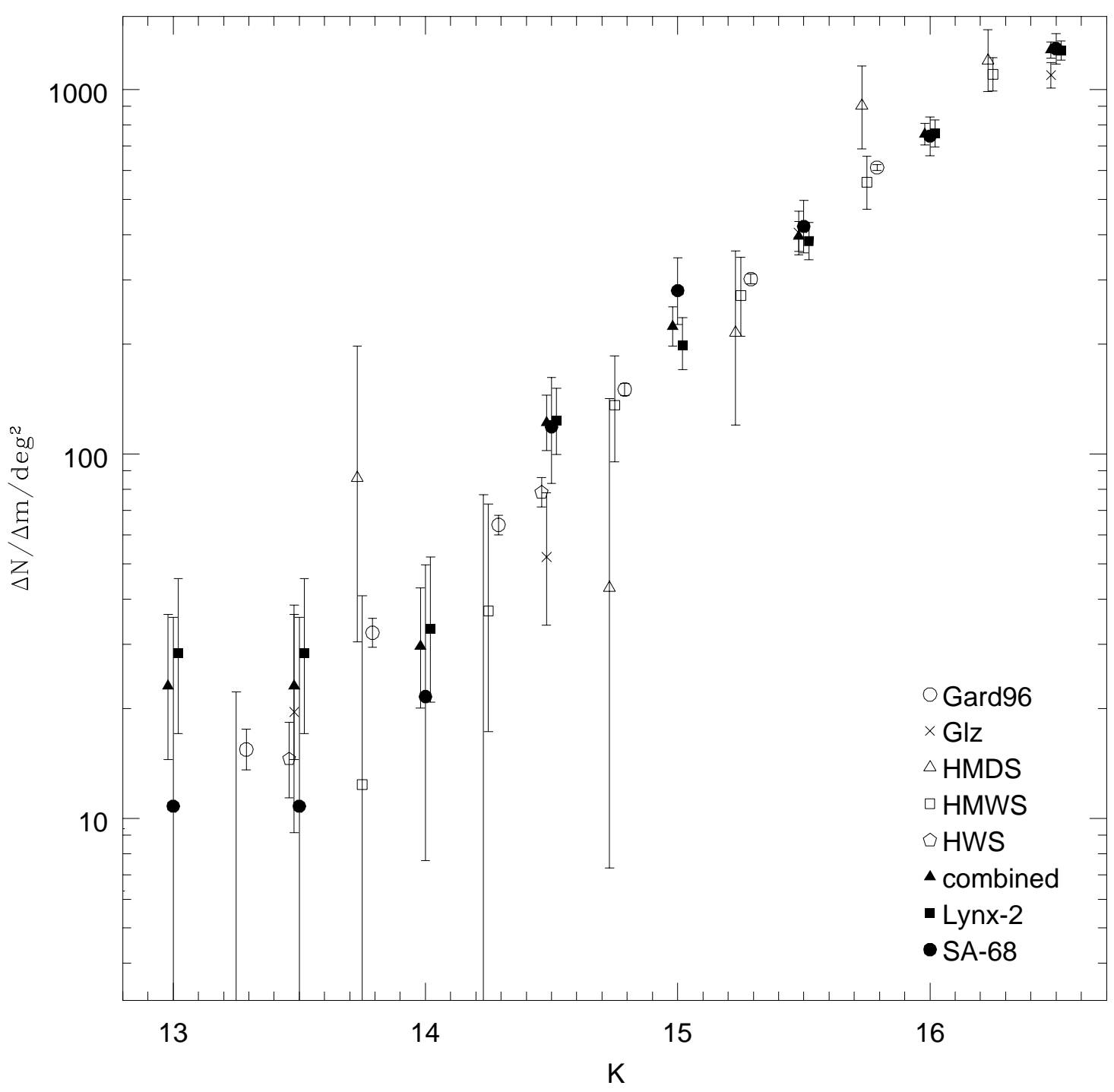

Fig. 4. - K-band differential number counts in the $K=13-16.5$ range. The data are a compilation from Gardner, Cowie and Wainscoat 1993 (HWS, HMWS, HMDS), Glazebrook et al. 1994 (Glz), Gardner 1996 (Gard96), our SA-68 and Lynx-2 fields, and our two fields combined (combined). Errors are $1 \sigma$ estimates from the raw counts. 

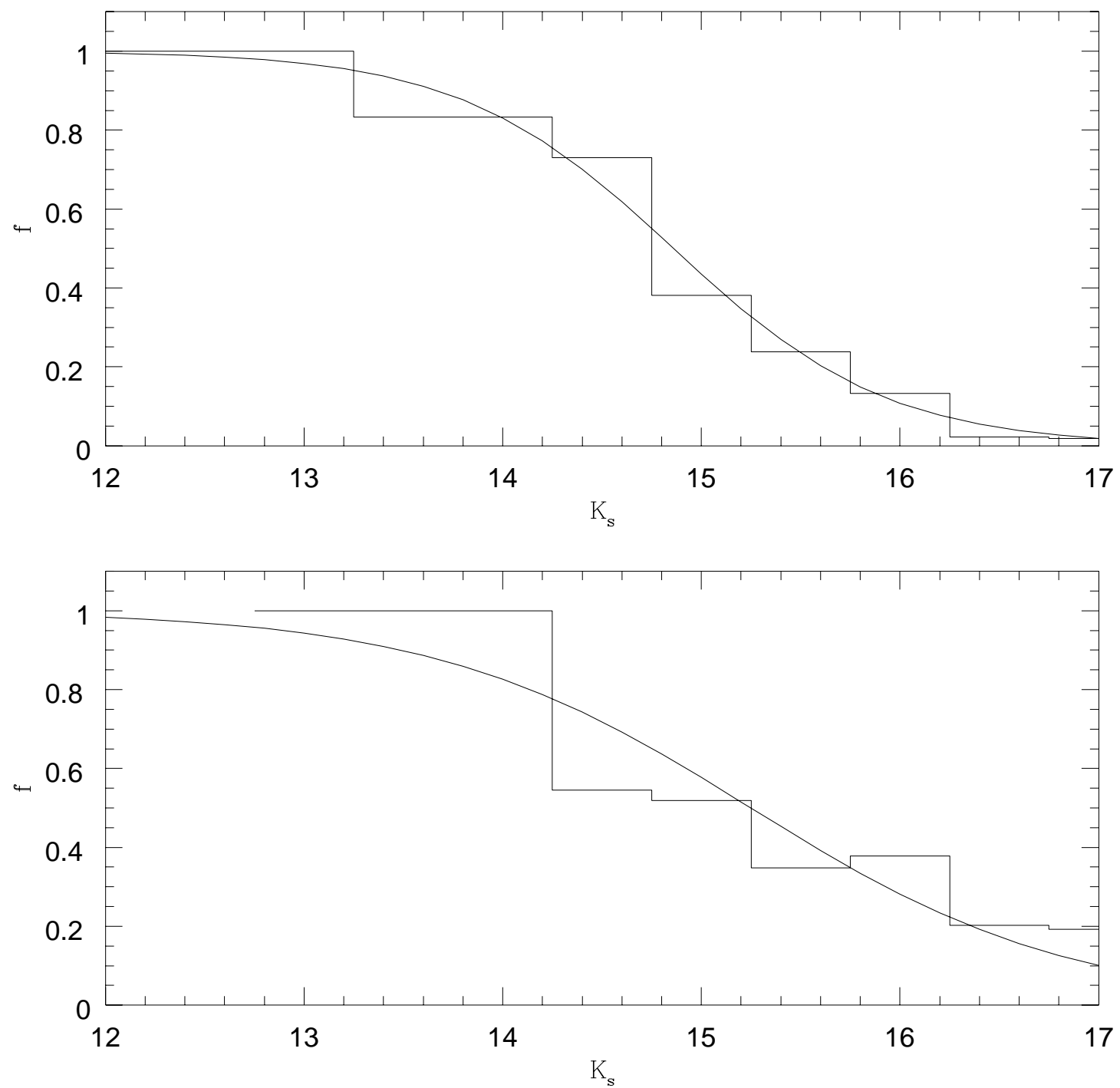

Fig. 5.- The redshift completeness, $f$, in each of the fields. The sample used in the luminosity function is cut at $K_{s}=16$ in SA68 and $K_{s}=15.25$ in Lynx2. Sandage functions are fit to the incompleteness with parameters, $m_{l}=15.25, \Delta m_{l}=0.80$ in SA68 and $m_{l}=14.86, \Delta m_{l}=0.54$ in Lynx2 (see text). 

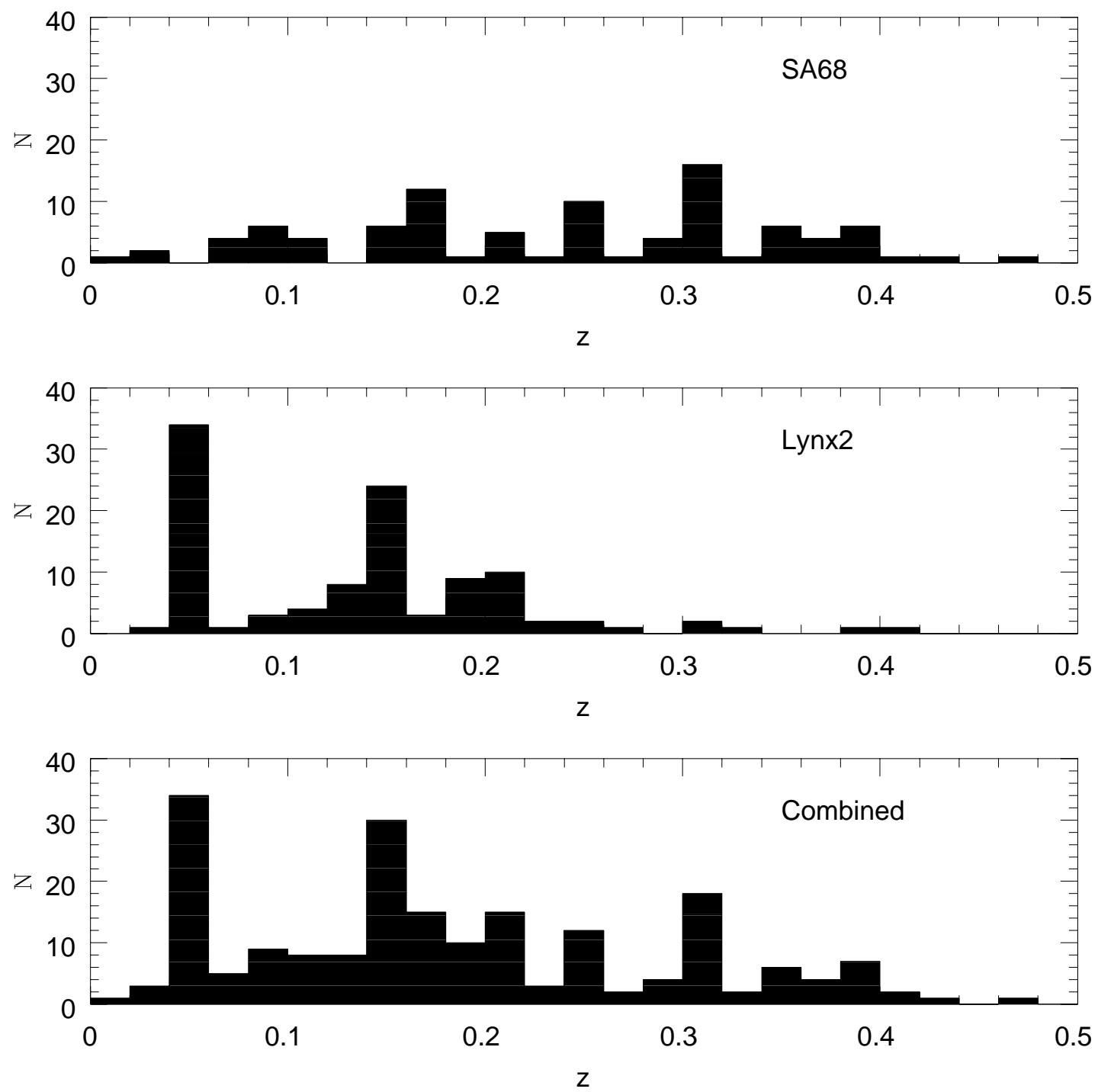

Fig. 6.- The redshift distribution of each sample of galaxies that were used in the luminosity function derivation. Two points are evident from these distributions. The majority of galaxies have redshifts $z<0.4$ and the Lynx2 field contains a low redshift cluster at $z=0.05$. The presence of significant structure in the redshift distribution requires the use of an estimator of the luminosity function that is not dependent on the clustering. 


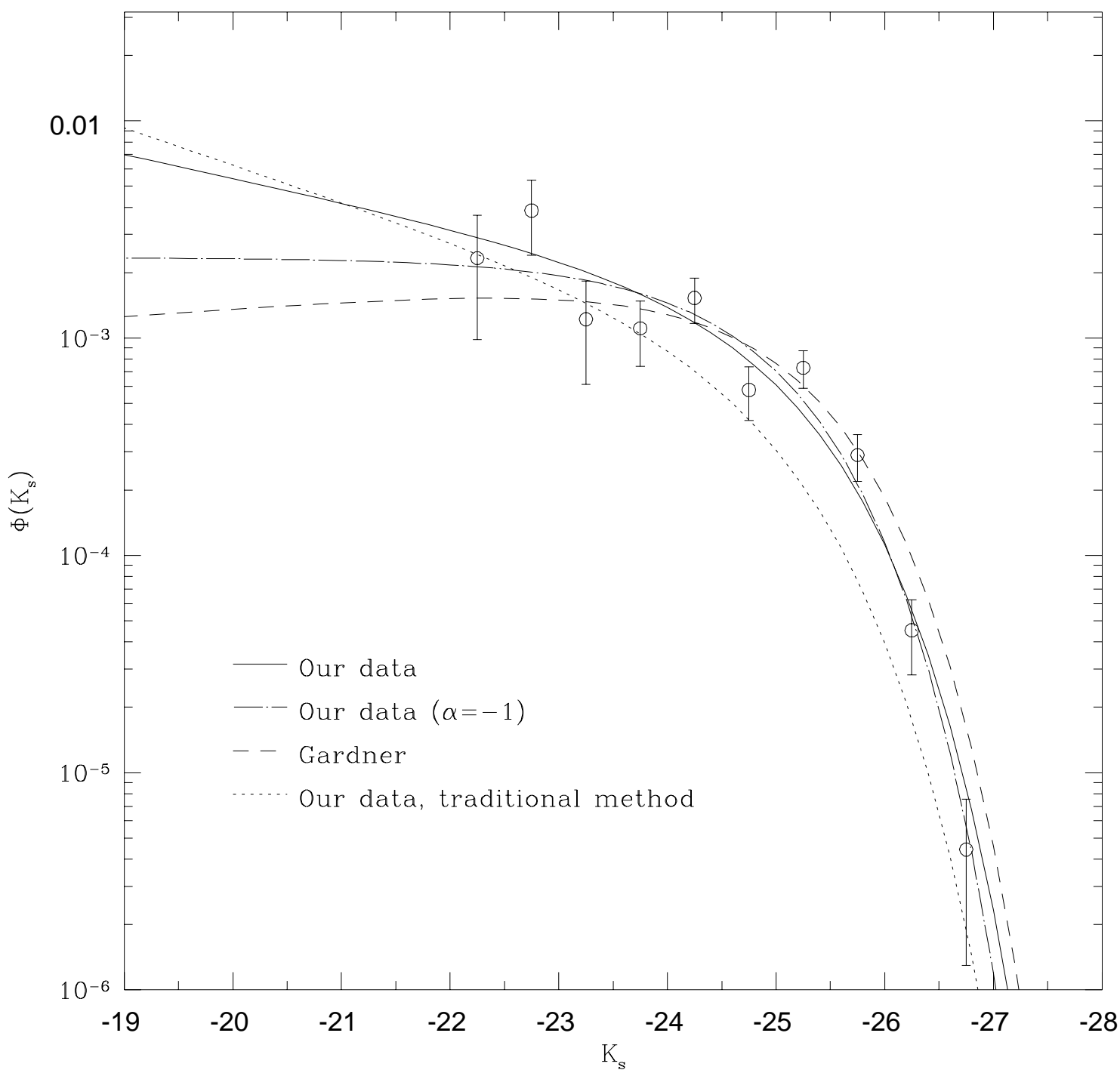

Fig. 7.- Differential luminosity function from our redshift sample. Error bars are calculated from the number of galaxies in each absolute magnitude bin, assuming Poisson statistics. The lines shown are for the Schechter functions fits (both with and without setting the parameter $\alpha$ to -1 ) as well as for previous determinations of the luminosity function. 


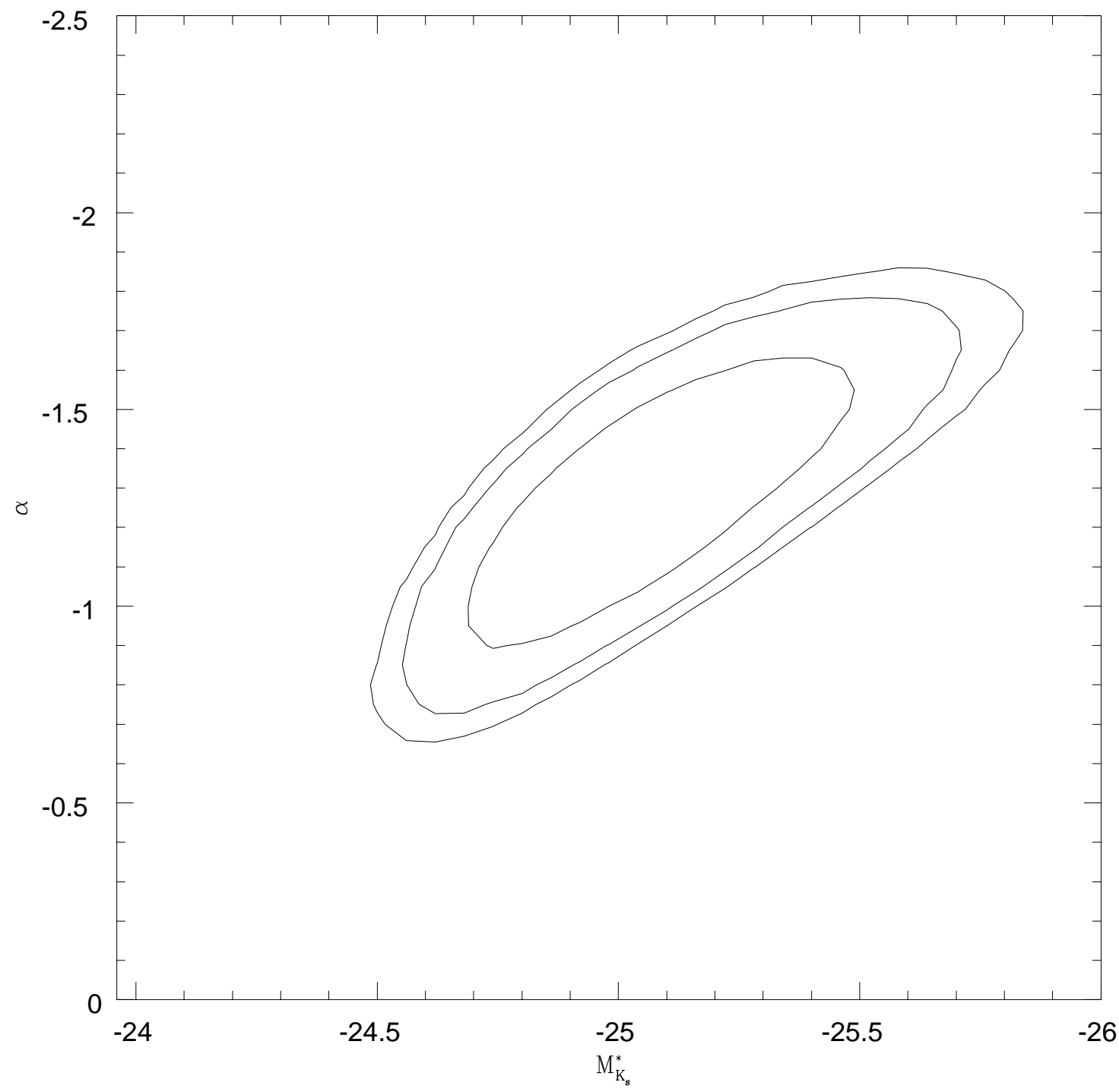

Fig. 8.- The confidence intervals for the Schechter parameters. The 68, 90 and 95 percentile confidence interval regions are shown derived from the maximum likelihood fitting techniques. 